\title{
Долинно-орбитальное взаимодействие в германии, легированном донорами V группы: количественный анализ
}

\author{
(C) А.А. Ревин ${ }^{1,2}$, А.М. Михайлова ${ }^{1,2}$, А.А. Конаков ${ }^{1,2, \uparrow}$, В.В. Цыпленков ${ }^{2}$, В.Н. Шастин ${ }^{2}$ \\ ${ }^{1}$ Национальный исследовательский Нижегородский государственный университет им. Н.И. Лобачевского, \\ 603950 Нижний Новгород, Россия \\ ${ }^{2}$ Институт ффизики микроструктур Российской академии наук, \\ 603950 Нижний Новгород, Россия \\ ๆ E-mail: konakov_anton@mail.ru
}

Поступила в Редакцию 12 апреля 2021 г.

В окончательной редакции 19 апреля 2021 г.

Принята к публикации 19 апреля 2021 г.

В рамках приближения огибающей функции рассчитаны волновые функции электронов, локализованных на мелких донорах $\mathrm{P}, \mathrm{As}, \mathrm{Sb}$ в Ge при учете долинно-орбитального взаимодействия, обусловленного короткодействующим потенциалом донора. Предложен подход, позволяющий включить междолинное смешивание в уравнение для многокомпонентной огибающей. Расчет эффектов долинно-орбитального взаимодействия проводился по теории возмущений, тогда как „затравочные“ однодолинные функции находились с применением вариационного метода Ритца. Параметры короткодействующей части потенциала и коэффициент междолинного смешивания подбирались индивидуально для каждого донора, позволяя получать наилучшее согласие с результатами экспериментального измерения энергий синглетного и триплетного состояний. Вычислены огибающие волновых функций состояний $1 s\left(A_{1}\right)$ и $1 s\left(T_{2}\right)$. Найдены параметры долинноорбитального взаимодействия для каждого донора. Также показано, как должны модифицироваться функции возбужденных состояний $2 s, 2 p_{0}, 2 p_{ \pm}, 3 p_{0}$, чтобы оставаться ортогональными функциям синглета и триплета в рамках более строгой многодолинной модели.

Ключевые слова: германий, мелкие доноры, долинно-орбитальное взаимодействие, приближение огибающей функции.

DOI: $10.21883 /$ FTP.2021.10.51441.40

\section{1. Введение}

Несмотря на долгую историю исследований [1], электронные свойства уединенных доноров замещения в кремнии и германии вызывают интерес и сейчас в связи с различными приложениями: для построения источников когерентного стимулированного излучения в ТГц частотном диапазоне [2,3], разработки электронных кубитов [4,5], квантовой памяти [6] и „одноатомных“ транзисторов [7]. В последние годы существенное внимание уделяется, в частности, изучению электронных свойств мелких доноров V группы в германии. Их энергии ионизации в 3-5 раз меныше, чем в кремнии [1], что позволяет, например, используя внутрицентровые переходы, получать более длинноволновое излучение, необходимое для решения ряда прикладных задач [8]. Доноры в германии проявляют на порядки бо́льшую, чем доноры в кремнии, нелинейную динамическую восприимчивость в дальнем ИК-диапазоне [9]. Кроме того, заметно (в $\sim 4$ раза) в сравнении с кремнием изменяется и масштаб волновых функций квантовых состояний мелких доноров в кристалле германия [1]. В таком случае открываются новые возможности воздействия на донорные состояния с помощью внешнего электрического поля [9] или размещая донор вблизи границы слоя германия [10]. Вместе с развитием технологии контролируемого на наномасштабах легирования герма- ния [11] указанные преимущества делают электронные и оптические свойства доноров замещения V группы в германии актуальным объектом исследования как с фундаментальной, так и с прикладной точек зрения.

Энергии электронных состояний доноров замещения V группы в германии хорошо известны из экспериментальных работ $[12,13]$ и могут быть проклассифицированы в соответствии с состояниями атома водорода: орбитально вырожденные возбужденные состояния $(2 p$ и выше) находятся в количественном согласии с теоретическими расчетами Фалкнера [14] в рамках метода эффективной массы, тогда как основное $1 s$ coстояние расщепляется в соответствии с тетраэдрической симметрией локального окружения на синглет $1 s\left(A_{1}\right)$ и триплет $1 s\left(T_{2}\right)[15]$ вследствие долинно-орбитального взаимодействия [16], обусловленного потенциалом „центральной ячейки“ (короткодействующей частью примесного потенциала) [17]. В то время как экспериментальные данные являются достаточно надежным источником информации об энергетических спектрах доноров, анализ вероятностей внутрицентровых излучательных и безызлучательных переходов $[18,19]$ и количественное предсказание внешнего воздействия на доноры, способного повысить квантовую эффективность генерации ТГц фотонов, требует расчета волновых функций локализованных носителей как в основном, так и в возбужденных состояниях. 
Ввиду большого по сравнению с параметром решетки германия $\left(a_{\mathrm{Ge}}=0.566 \mathrm{нм}\right)$ пространственного масштаба волновых функций электронов, локализованных на мелких донорах в германии ( $\sim 10$ нм в основном состоянии $[14,20])$, приближение огибающей функции становится наиболее оптимальным с точки зрения соотношения точности и вычислительной „стоимости“ методом расчета электронных состояний. Использование этого подхода для вычисления волновых функций доноров $\mathrm{V}$ группы в германии восходит к работам Киттеля и Митчелла [21] и Кона и Латтинжера [22]. В частности, последними впервые предложена система ортогональных функций, описывающих не только основное $1 s$, но и несколько возбужденных состояний доноров замещения в непрямозонных полупроводниках. Кроме того, в работе [22] отмечена необходимость учета междолинного взаимодействия для описания расщепления $1 s$ состояния доноров. Пантелидес и $\mathrm{Ca}[17]$ предложили модификацию метода огибающей, позволяющую количественно учитывать смешивание состояний различных энергетических минимумов в зоне проводимости (долинноорбитальное взаимодействие).

В нашей предыдущей работе [23] с применением приближения огибающей функции и вариационного метода Ритца были рассчитаны волновые функции низколежащих состояний $1 s\left(A_{1}\right), 2 s, 2 p_{0}, 2 p_{ \pm}, 3 p_{0}$ мелких донорных центров $\mathrm{P}, \mathrm{As}, \mathrm{Sb}$ в германии с учетом короткодействующего потенциала примеси. Каждая примесь характеризовалась своим полем, что позволило различить волновые функции для каждой примеси и получить зависимость энергий возбужденных состояний от типа примеси. Однако построенная модель была ограничена однодолинным приближением, которое обладает высокой точностью для возбужденных состояний [14], но не позволяет ввести в рассмотрение триплетный уровень $1 s\left(T_{2}\right)$, а также описать долинно-орбитальное расщепление $1 s$ состояния [17]. В новой работе ранее предложенный метод развит с учетом многодолинности зоны проводимости германия, в частности, выполнен расчет волновых функций синглета и триплета, согласованных с возбужденными водородоподобными состояниями, для доноров замещения $\mathrm{P}, \mathrm{As}, \mathrm{Sb}$.

\section{2. Гамильтониан задачи}

В задаче об электронных состояниях, формируемых замещением атома германия донором $\mathrm{V}$ группы, исходным является одноэлектронный гамильтониан вида

$$
\hat{H}_{0}(\mathbf{r})=\frac{\hat{\mathbf{p}}^{2}}{2 m_{0}}+V_{0}(\mathbf{r})+U(\mathbf{r})
$$

где $m_{0}-$ масса свободного электрона, $V_{0}(\mathbf{r})-$ периодический потенциал кристаллической структуры (идеального кристалла германия), обладающий пространственной симметрией $O_{h}^{7}$ (по Шенфлису), а $U(\mathbf{r})-$ потенциал, создаваемый заменой атома матрицы на атом приме- си, характеризуемый точечной тетраэдрической симметрией. Спин-орбитальное взаимодействие незначительно модифицирует состояния в зоне проводимости германия [24], поэтому пренебрегаем им в выражении (1).

Функция $U(\mathbf{r})$ определяется совокупностью трех факторов: различием в структуре ионных остовов донора и германия, деформацией ближайшего окружения, вносимого донором, а также экранированием примесного поля системой валентных электронов кристалла [17]. Последнее в однородной среде описывается статической диэлектрической функцией, которая в длинноволновом пределе переходит в статическую проницаемость $\varepsilon_{s}=16.0$ [25]. В результате потенциал $U(\mathbf{r})$ может быть представлен совокупностью двух слагаемых: дальнодействующим вкладом $U_{1-r}(\mathbf{r})$, доминирующим на масштабax, много бо́льших размера элементарной ячейки, в которой находится донор, и короткодействующим $U_{s h-r}(\mathbf{r})$, существенным на масштабе элементарной ячейки, содержащей донор. Дальнодействующий потенциал имеет водородоподобный вид

$$
U_{l-r}(\mathbf{r})=-\frac{e_{0}^{2}}{\mathcal{E}_{s}\left|\mathbf{r}-\mathbf{R}_{0}\right|},
$$

где $\mathbf{R}_{0}-$ радиус-вектор донора в кристалле, $e_{0}$ элементарный заряд.

В работе [23] нами была предложена следующая форма короткодействующего потенциала:

$$
\begin{aligned}
U_{s h-r}(\mathbf{r})= & -\frac{G e_{0}^{2}}{\left|\mathbf{r}-\mathbf{R}_{0}\right|}\left(B \exp \left(-\eta\left|\mathbf{r}-\mathbf{R}_{0}\right|\right)\right. \\
& \left.+\left(1-B-\frac{1}{\varepsilon_{s}}\right) \exp \left(-\kappa\left|\mathbf{r}-\mathbf{R}_{0}\right|\right)\right),
\end{aligned}
$$

где $G, B, \eta$ и $\kappa-$ феноменологические параметры, индивидуальные для каждой примеси. Структура потенциала (3) подобрана таким образом, чтобы в пределе водородоподобного центра с зарядом $+G e_{0}$ (в отсутствие эффектов, связанных с другой структурой внутренних оболочек примесных центров (nonisocoric impurity centers)) выражение (3) переходило в потенциал точечного заряда, статически экранированного с диэлектрической функцией, рассчитанной в работе [26]. Параметр $G$ играет роль эффективного заряда ионного остова, на который реагирует электронная подсистема кристалла вблизи примесного центра, а $B, \eta$, и $\kappa$ описывают затухание короткодействующего потенциала при удалении от примеси и коррелируют с ковалентным радиусом примесного центра. Обратим внимание, что потенциал в форме (3) не обладает тетраэдрической симметрией. Это обусловлено тем, что статическая диэлектрическая функция германия при расчетах была аппроксимирована сферически-симметричным выражением. В то же время различия в диэлектрической функции вдоль различных направлений (на малых масштабах), обусловленные анизотропией кристалла, составляют лишь несколько процентов [27]. Эта поправка несущественна в контексте 
точности, закладываемой нами в расчет, поэтому пренебрежем отличием потенциала от изотропного.

При гелиевых температурах ширина запрещенной зоны в германии составляет $\sim 0.74$ эВ [28], в то время как экспериментально установленные энергии ионизации мелких доноров - 0.012 эВ [1], поэтому волновые функции связанных состояний определяются в первую очередь блоховскими функциями минимума зоны проводимости (в точках $L$ зоны Бриллюэна) и в рамках приближения огибающей могут быть записаны в виде

$$
\Psi(\mathbf{r})=\sum_{j} \alpha_{j} F_{j}(\mathbf{r}) \varphi_{j}(\mathbf{r}),
$$

где $\varphi_{j}(\mathbf{r})$ - блоховские функции состояний четырех энергетических минимумов долинно-вырожденного дна зоны проводимости германия (нормированные на объем кристалла $\Omega), F_{j}(\mathbf{r})$ - отвечающие им компоненты огибающей (нормированные на единицу) в координатном представлении, $\alpha_{j}$ - коэффициенты разложения по состояниям различных долин, удовлетворяющие условию

$$
\sum_{j}\left|\alpha_{j}\right|^{2}=1
$$

Минимум зоны проводимости германи четырехкратно вырожден, а соответствующие неэквивалентным (не связанным векторами обратной решетки) точкам $L$ блоховские функции обозначим как $\varphi_{[111]}(\mathbf{r}), \varphi_{[11 \overline{1}]}(\mathbf{r}), \varphi_{[1 \overline{1} \overline{1}]}(\mathbf{r})$ и $\varphi_{[\overline{1} 1 \overline{1}]}(\mathbf{r})$ в соответствии с направлениями в обратном пространстве, на которых расположены эти точки.

Действуя оператором (1) на волновую функцию (4) и усредняя по быстро осциллирующим блоховским состояниям, можно получить эквивалентную систему уравнений для компонент огибающей $F_{j}(\mathbf{r})$ в рамках многодолинного приближения эффективной массы $[17,29]$. В блоховском базисе минимумов зоны проводимости эта система будет эквивалентна уравнению Шредингера с эффективным четырехрядным матричным гамильтонианом:

$$
\hat{H}_{i j}=\hat{H}_{i} \delta_{i j}+\hat{V}_{i j},
$$

где символ Кронекера $\delta_{i j}$ выделяет диагональную часть с элементами $\hat{H}_{i}, \hat{V}_{i j}$ - недиагональные элементы, а индексы $i$ и $j$,пробегают“ символические обозначения [111], [11 $\overline{1}],[1 \overline{1} \overline{1}]$ и $[\overline{1} 1 \overline{1}]$.

Диагональные элементы гамильтониана (6) могут быть записаны как

$$
\hat{H}_{i}=\frac{\hat{p}_{x}^{2}+\hat{p}_{y}^{2}}{2 m_{\perp}}+\frac{\hat{p}_{z}^{2}}{2 m_{\|}}+U_{l-r}(\mathbf{r})+\Omega\left|\varphi_{i}\left(\mathbf{R}_{0}\right)\right|^{2} U_{s h-r}(\mathbf{r}),
$$

где $m_{\|}=1.588 m_{0}$ и $m_{\perp}=0.0815 m_{0}$ [30] - продольная и поперечная эффективные массы вблизи дна зоны проводимости германия соответственно, причем ось $z$ декартовой системы координат ориентирована вдоль большой оси изоэнергетического эллипсоида. В выражении (7) $\left|\varphi_{i}\left(\mathbf{R}_{\mathbf{0}}\right)\right|^{2}$ представляет собой квадрат модуля блоховской функции $i$-го энергетического минимума в точке $\mathbf{R}_{0}$ расположения примесного центра. Недиагональные элементы $\hat{V}_{i j}$ определяются только короткодействующим потенциалом $U_{s h-r}(\mathbf{r})$ :

$$
\hat{V}_{i j}=\Omega \varphi_{i}^{+}\left(\mathbf{R}_{0}\right) \varphi_{j}\left(\mathbf{R}_{0}\right) U_{s h-r}(\mathbf{r}) .
$$

В выражении (6) именно элементы $\hat{V}_{i j}$ описывают долинно-орбитальное взаимодействие.

Основное отличие (7) и (8) от ранее использованного в работе [23] эффективного гамильтониана заключается в возникновении множителей вида $\Omega \varphi_{i}^{+}\left(\mathbf{R}_{0}\right) \varphi_{j}\left(\mathbf{R}_{0}\right)$ перед слагаемым $U_{s h-r}(\mathbf{r})$. Появление этих множителей обусловлено интегрированием блоховских функций с короткодействующим на масштабе элементарной ячейки потенциалом [29]. Фактически, если дальнодействующий потенциал на масштабе элементарной ячейки остается практически неизменным, то короткодействующий наоборот - изменяется драматически, так что существенными оказываются значения блоховских функций в точке расположения дефекта. Так как $\varphi_{j}(\mathbf{r}) \propto \frac{1}{\sqrt{\Omega}}$, то величины $\Omega \varphi_{i}^{+}\left(\mathbf{R}_{0}\right) \varphi_{j}\left(\mathbf{R}_{0}\right)$ безразмерны. Более того, они могут быть выбраны полностью действительными, так как относятся к вектору на границе зоны Бриллюэна. Значения $\varphi_{i}^{+}\left(\mathbf{R}_{0}\right) \varphi_{j}\left(\mathbf{R}_{0}\right)$ рассчитывались с использованием метода эмпирического псевдопотенциала [31] в базисе 169 плоских волн. Положение $\mathbf{R}_{0}$ соответствовало положению замещения в ячейке германия.

\section{3. Метод решения}

Уравнение Шредингера с матричным гамильтонианом (6) решалось последовательным применением вариационного метода Ритца и теории стационарных возмущений. В качестве невозмущенного гамильтониана использовалась диагональная часть оператора (6), т. е. фактически - однодолинное приближение, и эта задача решалась вариационным методом. В качестве функций нулевого приближения были выбраны линейные комбинации огибающих, соответствующие неприводимым представлениям $A_{1}$ и $T_{2}$ :

$$
\begin{aligned}
& A_{1}: F_{A}=\frac{1}{2}\left(F_{[111]} F_{[11 \overline{1}]} F_{[1 \overline{1} \overline{1}]} F_{[\overline{1} 1 \overline{1}]}\right) ; \\
& F_{1}=\frac{1}{2}\left(F_{[111]} F_{[11 \overline{1}]}-F_{[1 \overline{1} \overline{1}]}-F_{[\overline{1} 1 \overline{1}]}\right) ; \\
& T_{2}: F_{2}=\frac{1}{2}\left(F_{[111]}-F_{[11 \overline{1}]} F_{[1 \overline{1} \overline{1}]}-F_{[\overline{1} 1 \overline{1}]}\right) \text {; } \\
& F_{3}=\frac{1}{2}\left(F_{[111]}-F_{[11 \overline{1}]}-F_{[1 \overline{1} \overline{1}]} F_{[\overline{1} 1 \overline{1}]}\right) .
\end{aligned}
$$

Для расчета энергии и огибающей функции мелкого донора в германии в качестве элементов столбцов (8) мы использовали четырехпараметрическую пробную 
функцию, которая в системе главных осей изоэнергетического эллипсоида выглядит следующим образом:

$$
\begin{aligned}
F_{j}(\mathbf{r})= & N_{0}\left(\exp \left(-\sqrt{x^{2}+y^{2}+(\beta / \gamma) z^{2}} / R_{1}\right)\right. \\
& \left.+C_{0} \exp \left(-\sqrt{x^{2}+y^{2}+(\beta / \gamma) z^{2}} / R_{2}\right)\right),
\end{aligned}
$$

где $\gamma=m_{\perp} / m_{\|}-$параметр анизотропии эффективного гамильтониана в однодолинном пределе, $R_{1}$ и $R_{2}$ имеют смысл эффективных радиусов волновой функции, один из которых по смыслу отражает реакцию на дальнодействующую составляющую потенциала, а второй на короткодействующую, $C_{0}-$ безразмерный „весовой“ множитель, определяющий „смесь“ этих вкладов, $N_{0}-$ нормировочный множитель, а $\beta$ - характеризует анизотропию огибающей функции.

Недиагональные элементы $\hat{V}_{i j}$ учитывались по теории возмущений. При этом за счет симметризации огибающих по представлениям синглета и триплета гамильтониан (6) диагонализуется в базисе (9).

\section{4. Результаты расчетов и их обсуждение}

Энергии состояний, на которые расщепляется четырехкратно вырожденный по долинам уровень, могут быть записаны в виде

$$
\left\{\begin{array}{l}
E_{T}=E^{(0)}+Q N_{0}^{2}, \\
E_{A}=E^{(0)}-3 Q N_{0}^{2},
\end{array}\right.
$$

где $E^{(0)}$ - положение примесного уровня в отсутствие долинно-орбитального взаимодействия, а параметр $Q$ определяет междолинное смешивание.

Набор эмпирических параметров, входящих в потенциал (3), подбирался для каждой примеси индивидуально таким образом, чтобы энергии (11) при расчете совпадали со значениями, известными из эксперимента. Непосредственно вычисляемыми из теории являются значения $E^{(0)}$ и $Q$, которые легко сопоставить с эмпирическими данными:

$$
\left\{\begin{array}{l}
E^{(0)}=\frac{3 E_{T}+E_{A}}{4}, \\
Q=\frac{E_{T}-E_{A}}{4 N_{0}^{2}}
\end{array}\right.
$$

Полученные таким образом характеристики короткодействующего потенциала приведены в табл 1. Важно отметить, что они существенно отличаются от полученных ранее в работе [23]. В частности, множитель $G$, характеризующий эффективный заряд ядра, положен равным единице: отщепление синглета от триплета достигается за счет долинно-орбитального взаимодействия, которое в свою очередь различно для разных примесей. В работе [23] величину отщепления (точнее -
Таблица 1. Характеристики короткодействующего потенциала доноров замещения V группы в германии

\begin{tabular}{c|c|c|c|c}
\hline Донор & $B$ & $\eta\left(a_{0}^{-1}\right)$ & $\kappa\left(a_{0}^{-1}\right)$ & $G$ \\
\hline \multirow{2}{*}{$\mathrm{Ge}: \mathrm{P}$} & 0.47 & 0.42 & 0.41 & 1 \\
& $3.4^{*}$ & $0.62^{*}$ & $1.20^{*}$ & $1.27^{*}$ \\
\hline \multirow{2}{*}{$\mathrm{Ge}: \mathrm{As}$} & 0.47 & 0.40 & 0.41 & 1 \\
& $3.3^{*}$ & $0.61^{*}$ & $1.22^{*}$ & $1.32^{*}$ \\
\hline \multirow{\mathrm{Ge}}{*}{$\mathrm{Sb}$} & 0.47 & 0.49 & 0.48 & 1 \\
& $0.3^{*}$ & $0.78^{*}$ & $0.86^{*}$ & $4.39^{*}$
\end{tabular}

Примечание. * А.А. Ревин, А.М. Михайлова, А.А. Конаков, В.Н. Шастин. ФТП, 54, 938 (2020).

понижение энергии основного состояния в сравнении с водородоподобной моделью) существенно определял коэффициент $G$. В более полной многодолинной модели он, по всей видимости, оказывается избыточным. Параметр $B$ имеет ограниченный интервал изменения для выполнения условий, накладываемых на потенциал (3), а получаемая в расчетах энергия не сильно чувствительна к изменению $B$, так что для всех примесей было выбрано одно значение, соответствующее середине допустимого интервала. В результате на основе двух эмпирических величин $E^{(0)}$ и $Q$ определялись два параметра короткодействующего потенциала $\eta$ и $\kappa$, характеризующие масштаб затухания потенциала. Обратим внимание на то, что их значения для разных примесей оказываются близкими (см. табл. 1).

На рисунке приведены зависимости короткодействующих потенциалов для различных доноров в совокупности с экранированным в длинноволновом пределе кулоновским потенциалом. Как видно из табл. 1 и рисунка, короткодействующие потенциалы для фосфора и мышьяка в германии различаются слабо, тогда как

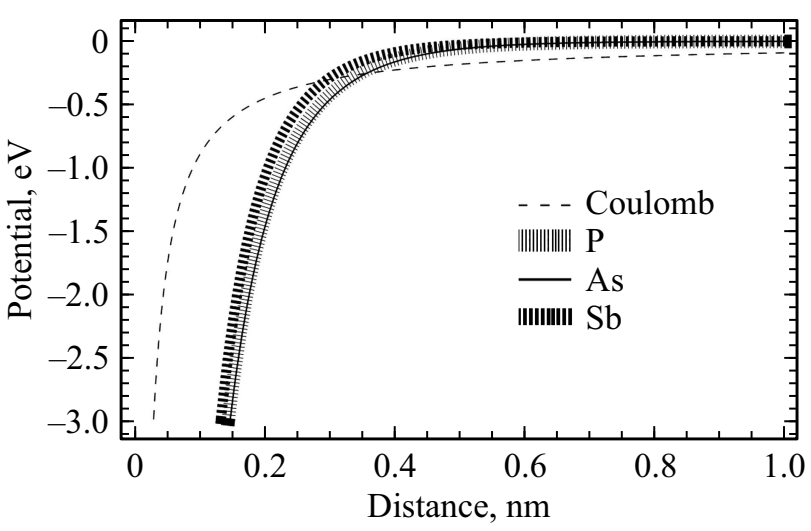

Потенциальная энергия электрона, локализованного на мелкой примеси в $\mathrm{Ge}$, в зависимости от расстояния до примесного центра. Отдельно выделен дальнодействующий кулоновский вклад (штриховой линией) и короткодействующие вклады, относящиеся к различным примесям (P, As, $\mathrm{Sb})$. 
Таблица 2. Энергии и параметры огибающих функций невозмущенного состояния (без долинно-орбитального взаимодействия)

\begin{tabular}{l|c|c|c|c|c}
\hline Донор & $E^{(0)}\left(E_{\mathrm{R}}\right)$ & $R_{1}\left(a_{\mathrm{B}}\right)$ & $R_{1}\left(a_{\mathrm{B}}\right)$ & $C$ & $\beta$ \\
\hline $\mathrm{Ge}: \mathrm{P}$ & -2.48 & 0.51 & 0.044 & 1.19 & 0.48 \\
$\mathrm{Ge}: \mathrm{As}$ & -2.54 & 0.50 & 0.045 & 1.27 & 0.48 \\
$\mathrm{Ge}: \mathrm{Sb}$ & -2.33 & 0.55 & 0.040 & 0.91 & 0.46
\end{tabular}

Таблица 3. Значения параметра междолинного смешивания $Q$ для каждого донора

\begin{tabular}{l|c}
\hline Донор & $Q, R y^{*} \cdot a_{\mathrm{B}}^{3}$ \\
\hline $\mathrm{Ge}: \mathrm{P}$ & 0.0034 \\
$\mathrm{Ge}: \mathrm{As}$ & 0.0035 \\
$\mathrm{Ge}: \mathrm{Sb}$ & 0.0046
\end{tabular}

потенциал для сурьмы имеет существенное отличие. Можно сделать предположение о том, что такое различие появляется из-за разницы в ковалентных радиусах атомов и локального растяжения химических связей в ближайшем окружении, вносимого атомом сурьмы. В целом тот же вывод был сделан и в работе [23] при решении задачи в однодолинном приближении.

Результаты расчета огибающей функции в невозмущенной задаче с использованием пробной функции (9) приведены в табл. 2. Значения энергий приведены в единицах эффективной энергии Ридберга

$$
R y^{*}=\frac{m_{\perp} e_{0}^{4}}{2 \hbar^{2} \varepsilon^{2}} \approx 4.33 \text { мэВ, }
$$

значения параметров функции - в эффективных боровских радиусах донора $a_{\mathrm{B}}=\hbar^{2} \varepsilon_{s} / m_{\perp} e_{0}^{2} \approx 10.39 \mathrm{HM}$ $\left(a_{\mathrm{B}} \approx 18.36 a_{\mathrm{Ge}}\right)$. Как видно из табл. 2 , один из радиусов $\left(R_{1}\right)$ оказывается на порядок больше параметра решетки германия и фактически описывает поведение волновой функции на большом расстоянии от примесного центра, когда потенциал уже можно считать водородоподобным. Второй радиус $\left(R_{2}\right)$ имеет порядок постоянной решетки и вносит вклад в волновую функцию только на размерах примитивной ячейки. Доля вклада с малым радиусом увеличивается, если основное состояние залегает глубже по энергии (в ряду центров $\mathrm{Ge}: \mathrm{Sb}, \mathrm{Ge}: \mathrm{P}, \mathrm{Ge}: \mathrm{As}$ ).

По значениям параметров функций, приведенных в табл. 2, можно рассчитать параметры долинно-орбитального взаимодействия $Q$. Их значения приведены в табл. 3. В частности, в случае управляемого воздействия на донорную волновую функцию (посредством внешних полей, механических напряжений или формирования границы) этот параметр не изменится, так как определяется локальными эффектами. Однако огибающая этой волновой функции может измениться существенным образом, так что параметр $Q$ является мерой эффектов междолинного взаимодействия для доноров не только в объемном германии, но и в германиевых структурах, и может быть использован при оценке, например, эффекта Штарка для примесных уровней [32].

В работе [23] нами были рассчитаны не только основное $1 s\left(A_{1}\right)$ состояние, но и возбужденные $2 s, 2 p_{0}$, $2 p_{ \pm}, 3 p_{0}$ состояния. Для всех них расщепление уровней вследствие долинно-орбитального взаимодействия крайне мало, и его можно не учитывать. Важно, чтобы функции возбужденных состояний были ортогональны с функциями состояний $1 s\left(A_{1}\right)$ и $1 s\left(T_{2}\right)$. Это достигается посредством введения функций, ортогональных в „своих“ долинах функциям (10). В частности, $2 s$-состояние описывается однодолинной (в системе главных осей изоэнергетического эллипсоида) огибающей вида [23]:

$$
\begin{aligned}
F_{2 s}(\mathbf{r})= & N_{1}\left(\exp \left(-\sqrt{x^{2}+y^{2}+(\beta / \gamma) z^{2}} / R_{3}\right)\right. \\
& \left.+C_{1} \exp \left(-\sqrt{x^{2}+y^{2}+(\beta / \gamma) z^{2}} / R_{4}\right)\right) \\
& \times\left(1+\chi_{1} \sqrt{x^{2}+y^{2}+(\beta / \gamma) z^{2}}\right)
\end{aligned}
$$

где $R_{3}, R_{4}, C_{1}$ и $N_{1}$ имеют тот же смысл, что $R_{1}, R_{2}, C_{0}$ и $N_{0}$ в $(10), \chi_{1}$ - параметр, обеспечивающий ортогональность основному состоянию, а значение $\beta$ берется то же, что и в выражении (10). Однодолинные функции орбитально вырожденных состояний в системе главных осей, согласно данным работы [14], имеют вид

$$
F_{n l m}(\mathbf{r})=(\beta / \gamma)^{1 / 4} f_{n l m}\left(x, y,(\beta / \gamma)^{1 / 2} z\right),
$$

где $f_{n l m}(\mathbf{r})$ - волновые функции атома водорода с набором квантовых чисел $n l m$. Один и тот же параметр $\beta$ в выражениях (10), (13) и (14), характеризующий спектр донора (для каждого - свой параметр), гарантирует ортогональность функций. В случае орбитально вырожденных состояний достаточно выбрать пробные функции с единственным пространственным масштабом, так как ортогональность функциям $s$-состояний достигается за счет угловой зависимости функций (14).

\section{5. Заключение}

В данной работе в рамках приближения огибающей рассчитаны волновые функции электронов, связанных с мелкими донорами $\mathrm{P}, \mathrm{As}, \mathrm{Sb}$ в Ge при учете долинноорбитального взаимодействия, обусловленного короткодействующим потенциалом донора. Предложен подход, позволяющий принять во внимание междолинное смешивание в уравнении для многокомпонентной огибающей. Долинно-орбитальное расщепление рассчитано по теории возмущений, в то время как задача в однодолинном пределе решалась вариационным методом Ритца. Параметры короткодействующей части потенциала и коэффициент междолинного смешивания подбирались индивидуально для каждого донора, позволяя получить 
наилучшее согласие с результатами экспериментального измерения энергий синглетного и триплетного состояний. Вычислены огибающие волновых функций состояний $1 s\left(A_{1}\right)$ и $1 s\left(T_{2}\right)$. Найдены параметры долинно-орбитального взаимодействия для каждого донора. Также показано, как должны модифицироваться функции возбужденных состояний $2 s, 2 p_{0}, 2 p_{ \pm}, 3 p_{0}$, чтобы оставаться ортогональными функциям синглета и триплета в рамках более строгой многодолинной задачи.

Достоинство избранного подхода перед другими методами расчета состоит в получении аналитических выражений для огибающих функций основного и возбужденных состояний локализованных на донорах электронов. С их помощью могут быть рассчитаны матричные элементы операторов различных физических величин, которые определяют, например, темпы излучательных и безызлучательных переходов между синглетом, триплетом и возбужденными состояниями примеси.

\section{Финансирование работы}

Работа поддержана грантом РНФ 19-72-20163.

\section{Благодарности}

Авторы признательны В.А. Бурдову за интерес к работе и стимулирующие обсуждения.

\section{Конфликт интересов}

Авторы заявляют, что у них нет конфликта интересов.

\section{Список литературы}

[1] A.K. Ramdas, S. Rodriguez. Rep. Progr. Phys., 44, 1297 (1981).

[2] S.G. Pavlov, N. Deßmann, B. Redlich, A.F.G. van der Meer, N.V. Abrosimov, H. Riemann, R.Kh. Zhukavin, V.N. Shastin, H.-W. Hübers. Phys. Rev. X, 8, 041003 (2018).

[3] S.G. Pavlov, N. Deßmann, A. Pohl, R.Kh. Zhukavin, T.O. Klaassen, N.V. Abrosimov, H. Riemann, B. Redlich, A.F.G. van der Meer, J.-M. Ortega, R. Prazeres, E.E. Orlova, A.V. Muraviev, V.N. Shastin, H.-W. Hübers. APL Photonics, 5, 106102 (2020).

[4] T.F. Watson, B. Weber, Y.-L. Hsueh, L.C.L. Hollenberg, R. Rahman, M.Y. Simmons. Sci. Advances, 3, e1602811 (2017).

[5] Y. He, S.K. Gorman, D. Keith, L. Kranz, J.G. Keizer, M.Y. Simmons. Nature, 571, 371 (2019).

[6] S. Freer, S. Simmons, A. Laucht, J.T. Muhonen, J.P. Dehollain, R. Kalra, F.A. Mohiyaddin, F.E. Hudson, K.M. Itoh, J.C. McCallum, D.N. Jamieson, A.S. Dzurak, A. Morello. Quant. Sci. Technol., 2, 015009 (2017).
[7] M. Fuechsle, J.A. Miwa, S. Mahapatra, H. Ryu, S. Lee, O. Warschkow, L.C.L. Hollenberg, G. Klimeck, M.Y. Simmons. Nature Nanotechnol., 7, 242 (2012).

[8] N. Deßmann, S.G. Pavlov, V.N. Shastin, R.Kh. Zhukavin, V.V. Tsyplenkov, S. Winnerl, M. Mittendorff, N.V. Abrosimov, H. Riemann, H.-W. Hübers, Phys. Rev. B, 89, 035205 (2014).

[9] N.H. Le, G.V. Lanskii, G. Aeppli, B.N. Murdin. Light: Sci. Appl., 8, 64 (2019).

[10] J. Salfi, J.A. Mol, R. Rahman, G. Klimeck, M.Y. Simmons, L.C.L. Hollenberg, S. Rogge. Nature Materials, 13, 605 (2014).

[11] G. Scappucci, G. Capellini, W.M. Klesse, M.Y. Simmons. Nanoscale, 5, 2600 (2013).

[12] J.H. Reuszer, P. Fisher. Phys. Rev., 135, A1125 (1964).

[13] S.D. Seccombe, D.M. Korn. Solid State Commun., 11, 1539 (1972).

[14] R.A. Faulkner. Phys. Rev., 184, 713 (1969).

[15] H. Fritzsche. Phys. Rev., 120, 1120 (1960).

[16] A. Baldereschi. Phys. Rev. B, 1, 4673 (1970).

[17] S.T. Pantelides, C.T. Sah. Phys. Rev. B, 10, 621 (1974).

[18] Р.Х. Жукавин, К.А. Ковалевский, Ю.Ю. Чопорова, В.В. Цыпленков, В.В. Герасимов, П.А. Бушуйкин, Б.А. Князев, Н.В. Абросимов, С.Г. Павлов, Г.-В. Хьюберс, В.Н. Шастин. Письма ЖЭТФ, 110, 677 (2019).

[19] К.А. Ковалевский, Ю.Ю. Чопорова, Р.Х. Жукавин, Н.В. Абросимов, С.Г. Павлов, Н.-W. Hubers, В.В. Цыпленков, В.Д. Кукотенко, Б.А. Князев, В.Н. Шастин. ФТП, 54, 1145 (2020).

[20] G. Pica, B.W. Lovett. Phys. Rev. B, 94, 205309 (2016).

[21] C. Kittel, A.H. Mitchell. Phys. Rev., 96, 1488 (1954).

[22] W. Kohn, J.M. Luttinger. Phys. Rev., 98, 915 (1955).

[23] А.А. Ревин, А.М. Михайлова, А.А. Конаков, В.Н. Шастин. ФТП, 54, 938 (2020).

[24] P. Li, Y. Song, H. Dery. Phys. Rev. B, 86, 085202 (2012).

[25] R. Resta. Phys. Rev. B, 16, 2718 (1977).

[26] S.T. Pantelides, C.T. Sah. Phys. Rev. B, 10, 638 (1974).

[27] J.P. Walter, M.L. Cohen. Phys. Rev. B, 2, 1821 (1970).

[28] S. Zwerdling, B. Lax, L.M. Roth, K.J. Button. Phys. Rev., 114, 80 (1959).

[29] L. Resca, R. Resta, H.B. Shore. Phys. Rev. B, 25, 4031 (1982).

[30] B.W. Levinger, D.R. Frankl. J. Phys. Chem. Solids, 20, 281 (1961).

[31] L.R. Saravia, D. Brust. Phys. Rev., 176, 915 (1968).

[32] A.J. Sigillito, A.M. Tyryshkin, J.W. Beeman, E.E. Haller, K.M. Itoh, S.A. Lyon. Phys. Rev. B, 94, 125204 (2016).

Редактор А.Н. Смирнов 
Valley-orbit coupling in germanium doped with group V donors: quantitative analysis

\author{
A.A. Revin ${ }^{1,2}$, A.M. Mikhaylova ${ }^{1,2}$, A.A. Konakov ${ }^{1,2}$, \\ V.V. Tsyplenkov ${ }^{2}$, V.N. Shastin ${ }^{2}$ \\ ${ }^{1}$ National Research Lobachevsky \\ State University of Nizhny Novgorod, \\ 603950 Nizhny Novgorod, Russia \\ ${ }^{2}$ Institute for Physics of Microstructures, \\ Russian Academy of Sciences, \\ 603950 Nizhny Novgorod, Russia
}

\begin{abstract}
In the framework of the envelope function approximation, the wave functions of electrons localized at shallow donors $\mathrm{P}, \mathrm{As}, \mathrm{Sb}$ in $\mathrm{Ge}$ are calculated taking into account the valleyorbit coupling caused by the donor short-range potential. It is proposed an approach that makes it possible to include inter-valley mixing in the equation for a multi-component envelope function. The calculation of the effects of the valley-orbit interaction was carried out according to the perturbation theory, while the „bare“ single-valley functions were found using the Ritz method. The parameters of the short-range part of the potential and the coefficient of inter-valley mixing were found individually for each donor, making it possible to obtain the best agreement with the results of experimental measurements of the energies of the singlet and triplet states. The envelope functions of the $1 s\left(A_{1}\right)$ and $1 s\left(T_{2}\right)$ states are calculated. The parameters of the valley-orbit interaction are found for each donor. It is also shown how the functions of the excited $2 s, 2 p_{0}, 2 p_{ \pm}, 3 p_{0}$ states should be modified in order to remain orthogonal to the singlet and triplet functions within the framework of a more rigorous multivalley model.
\end{abstract}

